\title{
Minocycline attenuates pain by inhibiting spinal microglia activation in diabetic rats
}

\author{
JIN-SHAN SUN ${ }^{1 *}$, YU-JIE YANG ${ }^{2 *}$, YONG-ZHEN ZHANG $^{1}$, WEN HUANG $^{1}$, \\ ZHAO-SHEN LI ${ }^{1}$ and YONG ZHANG ${ }^{3}$ \\ ${ }^{1}$ Department of Gastroenterology, Changhai Hospital, The Second Military Medical University, Shanghai 200433; \\ ${ }^{2}$ Department of Ophthalmology, No. 474 Hospital of Chinese People's Liberation Army, Urumqi, Xinjiang 830013; \\ ${ }^{3}$ Department of Anatomy and K. K. Leung Brain Research Center, The Fourth Military Medical University, \\ Xi'an, Shaanxi 710032, P.R. China
}

Received February 6, 2014; Accepted March 26, 2015

DOI: $10.3892 / \mathrm{mmr} .2015 .3735$

\begin{abstract}
The mechanisms associated with diabetes-induced neuropathic pain are complex and poorly understood. In order to understand the involvement of spinal microglia activity in diabetic pain, the present study investigated whether minocycline treatment is able to attenuate diabetic pain using a rat model. Diabetes was induced using a single intraperitoneal injection of streptozotocin (STZ). Minocycline was then intrathecally administered to the rats. Paw withdrawal threshold (PWT) and paw withdrawal latency (PWL) were tested weekly. The expression of OX-42, Iba-1, phospho-p38 mitogen-activated protein kinase (MAPK), tumor necrosis factor- $\alpha$ (TNF- $\alpha$ ), interleukin-1 $\beta$ (IL-1 $\beta$ ) and inducible nitric oxide synthase (iNOS), were examined in the spinal cord in order to evaluate the activation of microglia. The present study demonstrated that rats with STZ-induced diabetes exhibited increased mean plasma glucose concentration, decreased mean body weight and significant pain hypersensitivity compared with control rats. PWT and PWL values of rats with STZ-induced diabetes increased following treatment with minocycline. No differences were observed in expression levels of the microglial activity markers (OX-42, Iba-1 and phospho-p38 MAPK) between rats with STZ-induced diabetes and control rats. However, TNF- $\alpha$,
\end{abstract}

Correspondence to: Dr Zhao-Shen Li, Department of Gastroenterology, Changhai Hospital, The Second Military Medical University, 168 Changhai Road, Shanghai 200433, P.R. China

E-mail: zhaoshenli888@126.com

Dr Yong Zhang, Department of Anatomy and K. K. Leung Brain Research Center, The Fourth Military Medical University, 169 Changle West Road, Xi'an, Shaanxi 710032, P.R. China

E-mail: immuzhy@fmmu.edu.cn

*Contributed equally

Key words: diabetic neuropathic pain, hyperalgesia, microglia, minocycline
IL-1 $\beta$ and iNOS expression levels were higher in rats with STZ-induced diabetes compared with control rats. Following treatment with minocycline markers of microglial activation, including cytokines and iNOS, were downregulated in rats with STZ-induced diabetes. The results of the present study indicated that minocycline treatment may inhibit spinal microglial activation and attenuate diabetic pain in rats with STZ-induced diabetes.

\section{Introduction}

Diabetes mellitus is a group of metabolic diseases characterized by hyperglycemia as a result of insulin secretion and/or activity impairment. Diabetic neuropathy is a common complication that affects sensory neurons, motor neurons and the autonomic nervous system. Diabetic nerve pain is one of the most common symptoms of diabetic neuropathy and is characterized by spontaneous pain, hyperalgesia and paresthesias (1). The mechanisms underlying diabetic pain are complex and involve multiple mechanisms, including oxidative and nitrosative stress, immune system activation and mitochondrial dysfunction $(2,3)$. Several lines of clinical and experimental evidence have indicated that neuro-inflammation is an important factor in the process of peripheral and central diabetic neuropathy, which has been associated with the elevation of pro-inflammatory cytokines, including tumor necrosis factor- $\alpha$ (TNF- $\alpha$ ) and interleukin-1 $\beta$ (IL-1 $\beta$ ) (4-6).

Microglia, which are the predominant resident types of immune cells involved in the nervous system, mediate and regulate multiple inflammatory processes and are associated with the inflammatory changes underlying diabetic neuropathy (7). Minocycline is a tetracycline-derived antibiotic that exhibits anti-inflammatory properties in the central nervous system (8). It has been used in a number of animal models of neuro-inflammation in which microglia are implicated. Studies using rat models of pain facilitation have demonstrated that minocycline treatment inhibits microglial activation and cytokine expression $(9,10)$.

Activated microglia have been shown to cause or exacerbate diabetic pain. The effects of minocycline on pain in rats 
with streptozotocin (STZ)-induced diabetes were investigated in the present study.

\section{Materials and methods}

Animals. A total of 4210 week-old male Sprague-Dawley rats (200-250 g) were provided by the Experimental Animal Center of the Fourth Military Medical University (license no. RD-2010-06). Animals were housed under standard laboratory conditions, maintained on a $12 \mathrm{~h}$ light-dark cycle with food and water ad libitum. The experimental protocols were approved by the Institutional Animal Ethics Committee of the Fourth Military Medical University (Xi'an, China).

Experimental diabetic model. Diabetes was induced in 30 rats by administering one dose of STZ (Sigma-Aldrich, St. Louis, MO, USA) prepared in citrate buffer ( $\mathrm{pH} 4.4,0.1 \mathrm{M}$; Abcam, Cambridge, MA, USA). Six rats were removed from the study, due to failure of induction. STZ $(65-\mathrm{mg} / \mathrm{kg})$ was injected intraperitoneally. Twelve age-matched control rats were administered an equal volume of citrate buffer. Forty-eight hours following the injection, diabetes was confirmed by collecting blood samples from the tail vein. Plasma glucose levels were estimated using a commercial blood glucose analyzer (Accusoft, Roche Diagnostics, Laval, QC, Canada). Rats with plasma glucose levels $>300 \mathrm{mg} / \mathrm{dl}$ during fasting $(12-16 \mathrm{~h})$ were considered to be diabetic and included in the study. Body weight and plasma glucose levels were recorded twice per week for the duration of the study.

Intrathecal (IT) catheter implantation and drug administration. IT catheters were implanted as described by LoPachin (11). Rats were anesthetized using isoflurane (2\% in oxygen; RWD Life Science, Shenzhen, China). The occipital muscles were separated, and the cisternal membrane was exposed. IT polyethylene catheters (catalogue no. PE-10; outer diameter $0.5 \mathrm{~mm}$, inner diameter $0.25-\mathrm{mm}$, BD Intramedic ${ }^{\mathrm{TM}}$ Polyethylene Tubing; BD Sciences, Franklin Lakes, NJ, USA) were inserted via an incision in the cisterna magna and advanced caudally $7-8 \mathrm{~cm}$ deep to the lumbar enlargement of the spinal cord. The incision site was closed in layers, and the catheter was fixed firmly under the skin and heat-sealed. Animals recovered for 3 days until further treatment. Rats that exhibited no signs of motor deficiency (hind limb paralysis and stiffness) were used in the study. Drugs were injected via the IT catheter with an initial volume of $10 \mu \mathrm{l} \mathrm{STZ} \mathrm{followed} \mathrm{by}$ $10 \mu \mathrm{l}$ of saline solution.

The rats with STZ-induced diabetes and control rats were treated with 10,50 or $100 \mu \mathrm{g} / \mathrm{kg}$ minocycline (Sigma-Aldrich) dissolved in $10 \mu \mathrm{l}$ saline, or saline vehicle injection. IT administration of the drug or vehicle was initiated 4 days following STZ injection and was repeated twice daily at consistent times for the following 31 days. Paw withdrawal threshold (PWT) and paw withdrawal latency (PWL) were measured $1 \mathrm{~h}$ prior to minocycline or saline administration.

Assessment of mechanical allodynia. PWT was measured using an up-down testing paradigm. Rats were placed in cages with mesh floors and covered with transparent plastic boxes. They acclimatized to their surroundings for a minimum of $30 \mathrm{~min}$ in a temperature-controlled room $\left(25^{\circ} \mathrm{C}\right)$ prior to being tested. Von Frey hairs (Stoelting, Kiel, WI, USA) in $\log$ increments of force $(0.38,0.57,1.23,1.83,3.66,5.93,9.13$, and $13.1 \mathrm{~g}$ ) were applied for $4-6 \mathrm{sec}$ to the region between the foot pads in the plantar surface of the hind paw. Abrupt paw withdrawal, licking and shaking were interpreted as positive responses.

Assessment of thermal hyperalgesia. In order to assess nociceptive responses to thermal stimuli, the rats were placed in plexiglass chambers (18 x $8 \times 8 \mathrm{~cm}$; RWD Life Science) and radiant heat $\left(49^{\circ} \mathrm{C}\right)$, produced by an analgesia meter (cat. no. 390G; IITC Life Science, Woodland Hills, CA, USA), was applied to the plantar surface of the test paw. A cut-off time of $20 \mathrm{sec}$ was used in order to prevent tissue damage. PWL from the radiant heat was recorded using a plantar test (Hargreaves' method) analgesia meter. Abrupt paw withdrawal, licking and shaking were interpreted as positive responses.

Western blot analysis. Rats were anesthetized using $5 \%$ isoflurane and then decapitated. Lumbar spinal cords were dissected at L4-L6. Samples were homogenized in modified radioimmunopreciptation assay buffer $(50 \mathrm{mM}$ Tris- $\mathrm{HCl}$, pH 7.4; $1 \%$ nonyl phenoxypolyethoxylethanol, $1 \mathrm{mM}$ ethylenediaminetetraacetic acid and $150 \mathrm{mM} \mathrm{NaCl}$ ) supplemented with protease inhibitor cocktail (Sigma-Aldrich) diluted $1: 10$ and $2 \mathrm{mM}$ phenylmethanesulfonylfluoride, $2 \mathrm{mM} \mathrm{NaF}$ and phosphatase inhibitor cocktail I and II were added (Sigma-Aldrich). Lysates were then centrifuged at $4^{\circ} \mathrm{C}$ for $20 \mathrm{~min}$ at $12,000 \mathrm{x} \mathrm{g}$. The supernatants were collected and total protein concentration was measured using a bicinchoninic acid kit (Pierce Biotechnology, Inc., Rockford, IL, USA). Protein extracts were separated using SDS-PAGE in 7\% Tris- $\mathrm{HCl}$ gels (Bio-Rad Laboratories, Hercules, CA, USA) and transferred to polyvinylidene difluoridemembranes (EMD Millipore, Billerica, MA, USA), which were subsequently blocked using blocking solution [5\% dry non-fat milk in tris-buffered saline with $0.1 \%$ Tween $20^{\circledR}$ (TBST)] for $1 \mathrm{~h}$. Membranes were incubated with rabbit anti-Iba-1 antibody (1:1,000; cat. no. 016-20001; Wako Chemicals USA, Inc., Richmond, VA, USA), rabbit anti-OX-42 antibody (1:500; cat. no. RA25012; Neuromics, Edina, MN, USA), rabbit anti-phospho-p38 MAPK antibody (1:500; cat. no. 9212; Cell Signaling Technology, Inc., Danvers, MA, USA), mouse anti-p38 mitogen-activated protein kinase (MAPK) antibody (1:500; cat. no. ab31828; Abcam), rabbit anti-TNF- $\alpha$ antibody (1:500; cat. no. 3707; Cell Signaling Technology, Inc.), rabbit anti-IL-1 $\beta$ antibody (1:5,000; cat. no. ab200478; Abcam), or rabbit anti-inducible nitric oxide synthase (iNOS) antibody (1:2,000; cat. no. PA3-030A; Thermo Fisher, Rockford, IL, USA) in blocking solution overnight at $4^{\circ} \mathrm{C}$, washed in TBST and then incubated with horseradish peroxidase-conjugated anti-rabbit (cat. no. A0545) or anti-mouse (cat. no. A9044) immunoglobulin G (1:1,000; Sigma-Aldrich, St. Louis, MO, USA) for $1 \mathrm{~h}$ at room temperature. Membranes were then washed with TBST followed by TBS and developed using enhanced chemiluminescence detection reagent (GE Healthcare, Bio-Sciences, Pittsburgh, PA, USA) prior to film exposure (Kodak, Rochester, NY, USA) a number of 
Table I. Body weight and blood glucose levels in rats of different groups.

\begin{tabular}{|c|c|c|c|c|}
\hline \multirow[b]{2}{*}{ Group } & \multicolumn{2}{|c|}{ Body weight (g) } & \multicolumn{2}{|c|}{ Blood glucose $(\mathrm{mg} / \mathrm{dl})$} \\
\hline & Day 0 & Day 14 & Day 0 & Day 14 \\
\hline Control & $220 \pm 13.1$ & $293 \pm 12.8$ & $116 \pm 13.1$ & $121 \pm 12.7$ \\
\hline MC & $226 \pm 13.6$ & $285 \pm 14.1$ & $116 \pm 17.5$ & $123 \pm 16.4$ \\
\hline DM & $240 \pm 15.7$ & $195 \pm 12.2^{\mathrm{a}}$ & $114 \pm 14.8$ & $473 \pm 28.6^{b}$ \\
\hline $\mathrm{DM}+\mathrm{MC}$ & $228 \pm 15.2$ & $197 \pm 16.9$ & $118 \pm 15.1$ & $425 \pm 24.2$ \\
\hline
\end{tabular}

${ }^{\mathrm{a}} \mathrm{P}<0.05$ and ${ }^{\mathrm{b}} \mathrm{P}<0.01$ vs. control. $\mathrm{DM}$, diabetes mellitus; MC, minocycline.

A

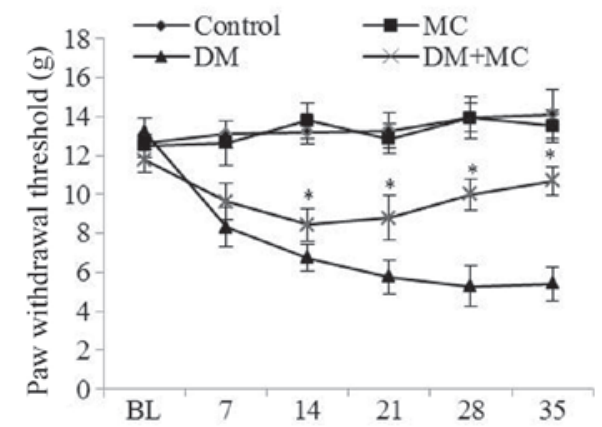

B

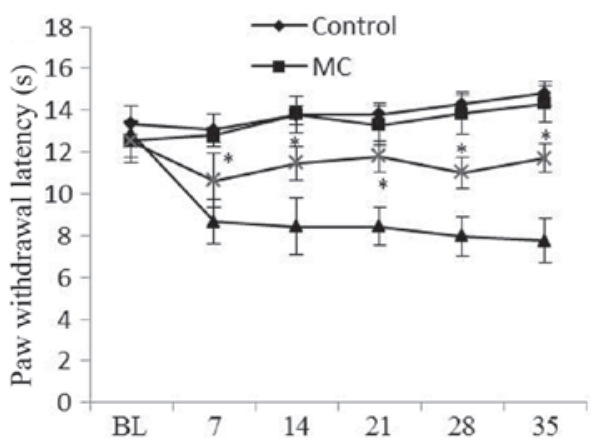

Figure 1. Minocycline treatment attenuates (A) mechanical allodynia and (B) thermal hyperalgesiain diabetic rats. MC, minocycline treated healthy rats; DM, diabetic rats with vehicle saline treatment; DM+MC, diabetic rats with minocycline treatment. ${ }^{*} \mathrm{P}<0.05$ vs. the control group; ${ }^{* *} \mathrm{P}<0.01$ vs. the DM group.

times. Membranes were re-probed with an antibody to $\beta$-actin for use as an internal loading control.

Statistical analysis. Quantitative data are expressed as the mean \pm standard error of the mean. Statistical significance among experimental groups was determined using one-way or two-way analysis of variance with Bonferroni multiple-comparison post-hoc analysis. $\mathrm{P}<0.05$ was considered to indicate a statistically significant difference. Statistical comparisons were computed using SigmaPlot 12.0 software (Systat Software, Inc., Chicago, IL, USA).

\section{Results}

Rats. Within the first week following STZ injection, rats demonstrated signs of diabetes, including weight loss, polydipsia and polyuria. Two weeks following STZ injection,
A

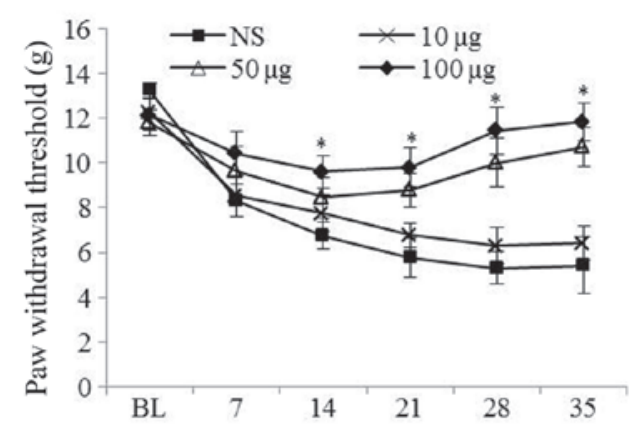

B

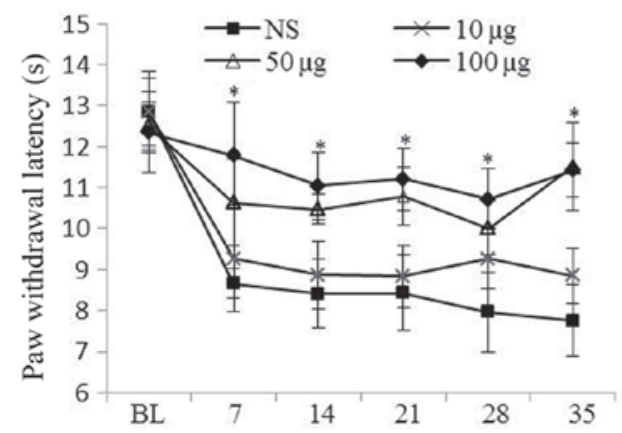

Figure 2. Minocycline attenuates mechanical allodynia in a dose-dependent manner (A) and thermal hyperalgesia (B) in diabetic rats. ${ }^{*} \mathrm{P}<0.05$ vs. saline vehicle injected rats. BL, baseline; NS, saline vehicle injected group.

rats exhibiting a glucose level $>300 \mathrm{mg} / \mathrm{dl}$ were included in the diabetic group. Rats with STZ-induced diabetes exhibited significantly increased blood glucose levels $(473 \pm 28.6 \mathrm{mg} / \mathrm{dl})$ compared with the control group $(121 \pm 12.7 \mathrm{mg} / \mathrm{dl})$ and reduced body weight $(195 \pm 12.2 \mathrm{~g})$ compared with the control group (293 $\pm 12.8 \mathrm{~g}$; Table I).

PWT values were significantly lower in diabetic rats than that in control rats, suggesting the presence of mechanical allodynia in diabetic rats. However, allodynia was reduced in diabetic rats treated with minocycline compared with those treated with vehicle (Fig. 1A). The threshold for thermal hyperalgesia was significantly decreased following STZ-injection compared with that in control rats. Similar to mechanical allodynia, hyperalgesia was significantly lower in diabetic rats treated with minocycline than in those treated with saline vehicle (Fig. 1B). Minocycline treatment attenuated mechanical allodynia and hyperalgesia in a dose-dependent manner. Minocycline treatment (50 and $100 \mu \mathrm{g}$ ) was shown to reduce 

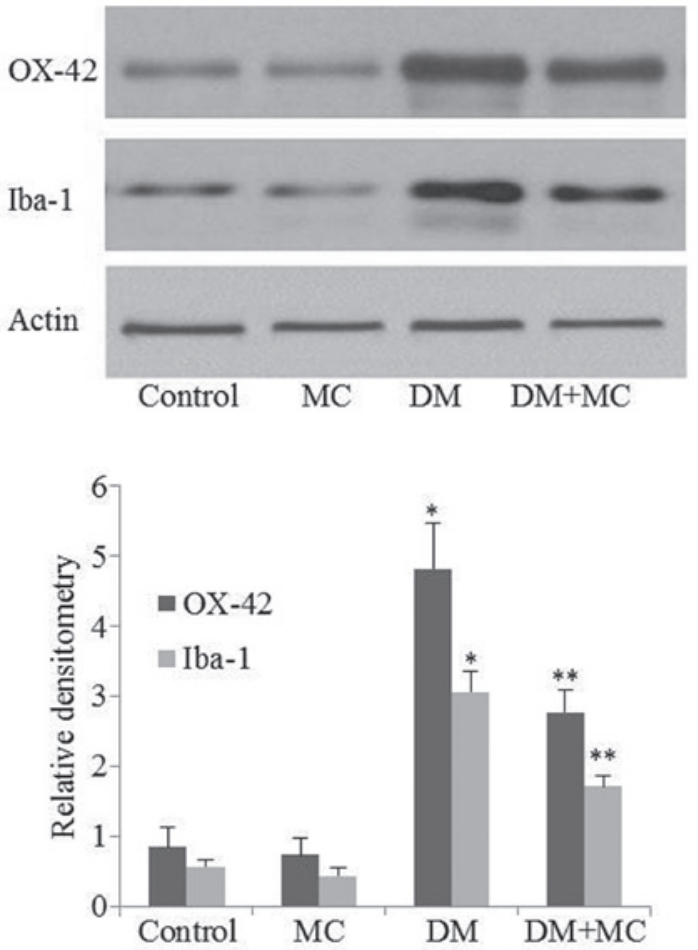

Figure 3. Expression of OX-42 and Iba-1 in the spinal cord dorsal horn was inhibited following minocycline treatment in diabetic rats. Immunoblotting of $\beta$-actin was used as a control. ${ }^{* * *} \mathrm{P}<0.01$ vs. the $\mathrm{DM}$ group and ${ }^{*} \mathrm{P}<0.05$ vs. the control group. DM, diabetic rats with saline vehicle treatment; $\mathrm{MC}$, minocycline.
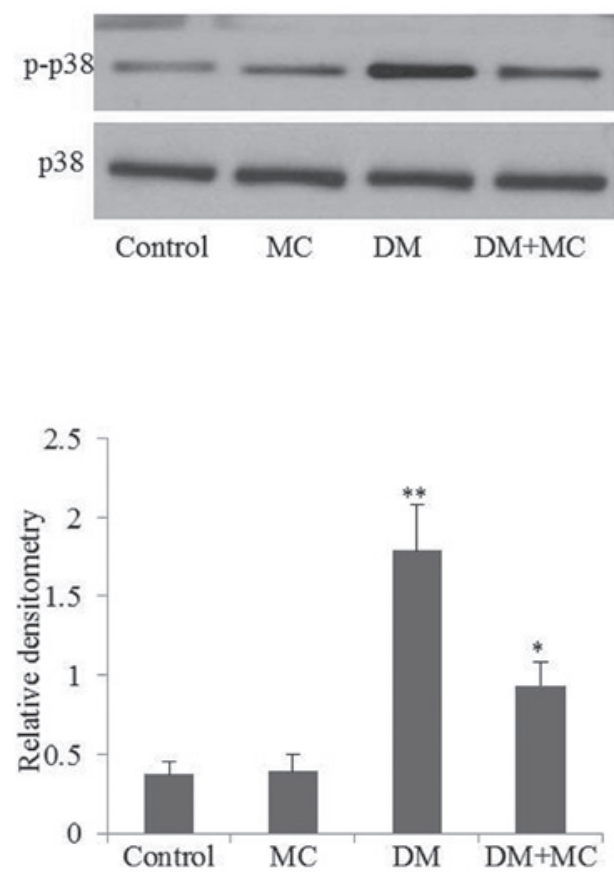

Figure 4. Phospho-p38 mitogen-activated protein kinase expression in spinal cord dorsal horn was downregulated following minocycline treatment of diabetic rats. ${ }^{*} \mathrm{P}<0.05$ vs. control group, ${ }^{* *} \mathrm{P}<0.01$ vs. the $\mathrm{DM}$ group and ${ }^{* * *} \mathrm{P}<0.01$ vs. the $\mathrm{DM}$ group. DM, diabetic rats with saline vehicle treatment; MC, minocycline.

diabetic pain in diabetic rats compared with those treated with vehicle $(\mathrm{P}<0.05$; Fig. 2A and $2 \mathrm{~B})$.
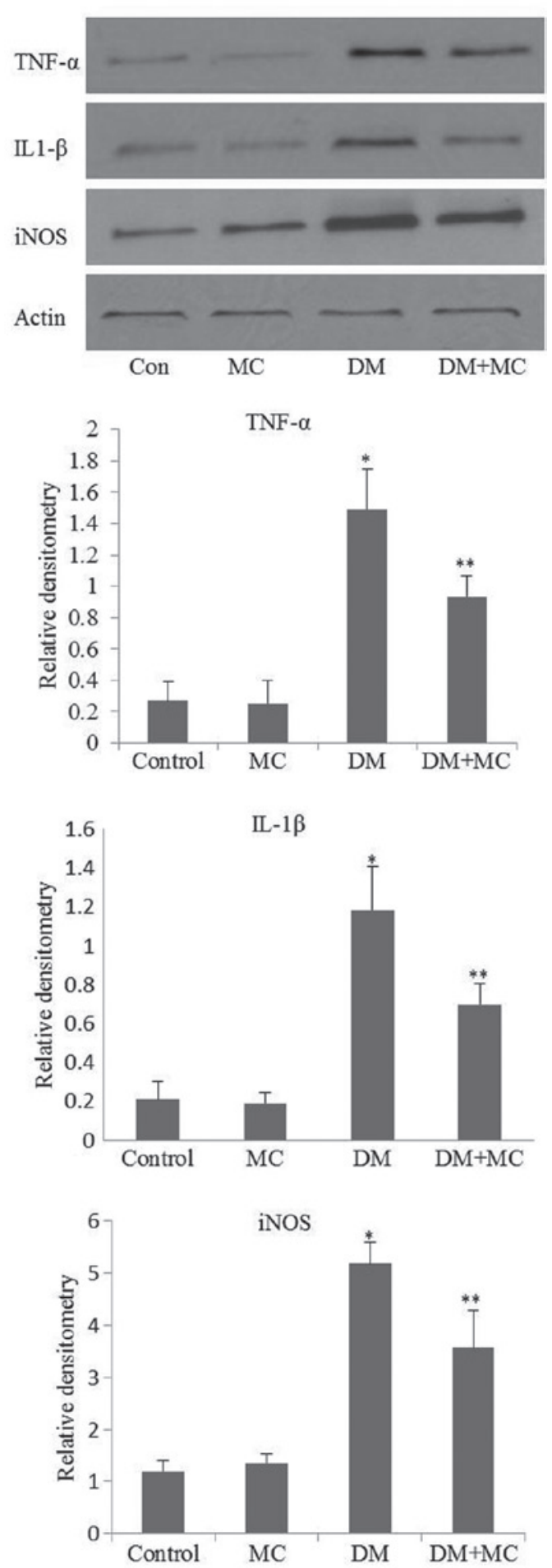

Figure 5. Expression of TNF- $\alpha$, IL-1 $\beta$ and iNOS in the spinal cord dorsal horn was inhibited following minocycline treatment in diabetic rats. Immunoblotting of $\beta$-actin was used as a control. ${ }^{*} \mathrm{P}<0.05$ vs. the control group and ${ }^{* *} \mathrm{P}<0.05$ vs. the DM group. TNF- $\alpha$, tumor necrosis factor- $\alpha$; IL- $\beta$, interleukin-1 $\beta$; iNOS, inducible nitric oxide synthase; DM, diabetic rats with saline vehicle treatment; MC, minocycline.

OX-42 and Iba-1 expression is upregulated in activated microglia. In order to assess the effects of minocycline on microglial activation in diabetic rats, OX-42 and Iba-1 expression was measured using western blotting. Western blotting suggested that OX-42 and Iba-1 expression levels 
were upregulated in diabetic rats compared with control rats. However, their expression levels in the spinal cord decreased significantly following minocycline treatment of diabetic rats compared with those treated with saline vehicle (Fig. 3; $\mathrm{P}<0.05$ ). The p38 MAPK signaling pathway is involved in microglial activation and cytokine production. Therefore, phospho-p38 MAPK levels were analyzed in the present study. The results demonstrated that phospho-p38 MAPK expression in diabetic rats was significantly higher compared with the control group (Fig. 4; P<0.05). However, the level of phospho-p38 MAPK expression decreased following minocycline treatment. The results suggested that microglia were activated in diabetic rats, while minocycline treatment of diabetic rats inhibited microglial activation.

Activated microglia secrete proinflammatory cytokines and $i N O S$, which are involved in pain hypersensitivity. The expression of TNF- $\alpha$, IL-1 $\beta$ and iNOS in the spinal cords of diabetic rats was significantly higher compared with that in the control group. However, following minocycline treatment, the expression levels of TNF- $\alpha$, IL-1 $\beta$ and iNOS were significantly lower in diabetic rats compared with those of the saline vehicle-treated group (Fig. 5; $\mathrm{P}<0.05$ ). Proinflammatory cytokines and iNOS expression was not completely inhibited in minocycline-treated diabetic rats compared with saline vehicle-treated diabetic rats. This may be explained by the presence of astrocytes, which are not inhibited by minocycline and may contribute to the expression of proinflammatory cytokines and iNOS (12).

\section{Discussion}

Inflammation is involved in the progression of diabetic neuropathic complications. Proinflammatory cytokines, such as TNF- $\alpha$ and IL-1 $\beta$ have been found to exhibit increased expression in diabetic patients (13). In chronic hyperglycemia, proinflammatory cytokines and reactive oxygen species (ROS) infiltrate vascular tissues and activate microglia. Recent studies have shown that microglia activation is associated with the initiation and maintenance of neuropathic pain $(14,15)$. Activated microglia release a number of neurotoxins that further enhance microglial proliferation and activation (10). Activated microglia excrete TNF- $\alpha$ and IL-1 $\beta$. In addition, via the expression of iNOS, activated microglia produce toxic mediators, such as ROS and nitric oxide (NO). Proinflammatory cytokines and chemokines are associated with hyperalgesia (5).

TNF- $\alpha$ expression induces the phosphorylation of c-Jun $\mathrm{N}$-terminal kinase 1 and activates nuclear factor $\kappa \mathrm{B}(\mathrm{NF}-\kappa \mathrm{B})$, leading to chemokine (C-C motif) ligand 2 (CCL2) release. CCL2 then acts on C-C chemokine receptor type 2 (CCR2) receptors on neurons and interacts positively with neuronal $\mathrm{N}$-methyl-D-aspartate (NMDA) and $\alpha$-Amino-3-hydroxy-5 -methyl-4-isoxazolepropionic acid (AMPA) receptors (16). In the rostral ventromedial medulla, which is responsible for maintaining chronic neuropathic pain, TNF- $\alpha$ is induced following nerve injury and facilitates NMDA receptor phosphorylation (17). TNF- $\alpha$ also stimulates phosphorylation of the glutamate A1 subunit of the AMPA receptor and promotes its trafficking to the membrane in dorsal horn neurons $(9,18)$.

IL-1 $\beta$ is another proinflammatory cytokine involved in pain hypersensitivity. The release of IL-1 $\beta$ is mediated by chemokine (C-X3-C motif) ligand 1 signaling and p38 MAPK activation, and is dependent on adenosine triphosphate. Following spinal nerve injury or inflammation, pro-IL-1 $\beta$ is cleaved by matrix metalloproteinase- 9 in microglia. IL-1 $\beta$ is an important messenger between glial cells and neurons. Activation of the IL-1 receptor causes it to colocalize with NMDA receptors, facilitating NMDA receptor phosphorylation, which induces changes in synaptic strength and results in hyperalgesic behavior $(17,19)$. However, IL-1 $\beta$ may also function in an NMDA receptor-independent manner (20).

ROS are associated with the development of persistent pain that results from nerve injury or inflammatory insult (21). Studies have shown that ROS in the spinal cord may induce pain by reducing the inhibitory effect of $\gamma$-aminobutyric acid on substantia gelatinosa neurons that are involved in pain transmission (22). High NO is associated with diabetic neuropathic pain (23). The production of NO occurs via increased iNOS activity following chronic inflammation in diabetic patients. There is evidence to suggest that there is a reciprocal correlation between $\mathrm{NO}$ and prostaglandin (PG) biosynthetic pathways (24). NO directly influences cyclooxygenase expression and PG biosynthesis (23).

In the present study, the PWT and PWL in diabetic rats were markedly lower compared with control rats. Spinal cord expression levels of OX-42, Iba-1 and phospho-p38 MAPK were elevated in diabetic rats compared with those in control rats. Microglia activation produced additional proinflammatory cytokines and toxic mediators, which are involved in diabetic pain. When treated with minocycline, diabetic rats exhibited reduced mechanical allodynia and thermal hyperalgesia compared with those treated with saline vehicle. The inhibition of microglial activation was confirmed by a decrease in the expression of microglia markers and proinflammatory cytokines. The results of the present study also demonstrated that the expression of proinflammatory cytokines and iNOS could not be completely blocked by treatment with minocycline. This was partly because astrocytes, which cannot be inhibited by minocycline, also contribute to the expression of proinflammatory cytokines and iNOS (12). These results suggested that minocycline may attenuate diabetic pain by inhibiting microglial activity.

The mechanisms underlying the influence of minocycline on diabetic rats are currently unclear. However, recent studies have suggested that minocycline inhibits MAPK- and $\mathrm{NF}-\kappa \mathrm{B}$-dependent signaling pathways in primary microglia and microglial cell cultures compared with controls (13). Furthermore, minocycline suppresses microglial expression of OX-42 and major histocompatibility complex class II in rat brains via a protein kinase C-dependent mechanism (15).

In conclusion, the results of the present study indicated a potential effect of minocycline for the treatment of diabetic hypersensitivity. Minocycline may inhibit spinal microglial activation and attenuate diabetic pain in diabetic rats. Therefore, further investigation into the use of microglial inhibition in the treatment of incurable diabetic pain may be beneficial.

\section{Acknowledgements}

The present study was supported by the National Natural Science Foundation of China (grant nos. 81100816 and 81000563). 


\section{References}

1. Yagihashi S, Yamagishi S and Wada R: Pathology and pathogenetic mechanisms of diabetic neuropathy: Correlation with clinical signs and symptoms. Diabetes Res Clin Pract 77 (Suppl 1): S184-S189, 2007.

2. Green CJ, Pedersen M, Pedersen BK and Scheele C: Elevated $\mathrm{NF}-\kappa \mathrm{B}$ activation is conserved in human myocytes cultured from obese type 2 diabetic patients and attenuated by AMP-activated protein kinase. Diabetes 60: 2810-2819, 2011.

3. Newsholme P, Gaudel C and Krause M: Mitochondria and diabetes. An intriguing pathogenetic role. Adv Exp Med Biol 942: 235-247, 2012

4. Zhang YL, Xu JM, Zhou P, Zhong XL and Dai RP: Distinct activation of tumor necrosis factor- $\alpha$ and interleukin- 6 in the spinal cord after surgical incision in rats. Mol Med Rep 5: 1423-1427, 2012.

5. Ren K and Dubner R: Interactions between the immune and nervous systems in pain. Nat Med 16: 1267-1276, 2010.

6. Jung WW, Kim HS, Shon JR, Lee M, Lee SH, Sul D, Na HS, Kim JH and Kim BJ: Intervertebral disc degeneration-induced expression of pain-related molecules: Glial cell-derived neurotropic factor as a key factor. J Neurosurg Anesthesiol 23: 329-334, 2011.

7. Prinz M, Tay TL, Wolf Y and Jung S: Microglia: Unique and common features with other tissue macrophages. Acta Neuropathol 128: 319-331, 2014

8. Amin AR, Attur MG, Thakker GD, Patel PD, Vyas PR, Patel RN, Patel IR and Abramson SB: A novel mechanism of action of tetracyclines: Effects on nitric oxide synthases. Proc Natl Acad Sci USA 93: 14014-14019, 1996.

9. Leung L and Cahill CM: TNF-alpha and neuropathic pain - a review. J Neuroinflammation 7: 27, 2010.

10. Naseri K, Saghaei E, Abbaszadeh F, Afhami M, Haeri A, Rahimi F and Jorjani M: Role of microglia and astrocyte in central pain syndrome following electrolytic lesion at the spinothalamic tract in rats. J Mol Neurosci 49: 470-479, 2013.

11. LoPachin RM, Rudy TA and Yaksh TL: An improved method for chronic catheterization of the rat spinal subarachnoid space. Physiol Behav 27: 559-561, 1981.

12. Ihara H, Yamamoto H, Ida T, Tsutsuki H, Sakamoto T, Fujita T, Okada T and Kozaki S: Inhibition of nitric oxide production and inducible nitric oxide synthase expression by a polymethoxyflavone from young fruits of Citrus unshiu in rat primary astrocytes. Biosci Biotechnol Biochem 76: 1843-1848, 2012.
13. Nikodemova M, Duncan ID and Watters JJ: Minocycline exerts inhibitory effects on multiple mitogen-activated protein kinases and IkappaBalpha degradation in a stimulus-specific manner in microglia. J Neurochem 96: 314-323, 2006.

14. Lim H, Kim D and Lee SJ: Toll-like receptor 2 mediates peripheral nerve injury-induced NADPH oxidase 2 expression in spinal cord microglia. J Biol Chem 288: 7572-7579, 2013.

15. Nikodemova M, Watters JJ, Jackson SJ, Yang SK and Duncan ID Minocycline down-regulates MHC II expression in microglia and macrophages through inhibition of IRF-1 and protein kinase $\mathrm{C}$ (PKC)alpha/betaII. J Biol Chem 282: 15208-15216, 2007.

16. Gao YJ, Xu ZZ, Liu YC, Wen YR, Decosterd I and Ji RR: The c-Jun N-terminal kinase 1 (JNK1) in spinal astrocytes is required for the maintenance of bilateral mechanical allodynia under a persistent inflammatory pain condition. Pain 148: 309-319, 2010.

17. Wei F, Guo W, Zou S, Ren K and Dubner R: Supraspinal glial-neuronal interactions contribute to descending pain facilitation. J Neurosci 28: 10482-10495, 2008.

18. Choi JI, Svensson CI, Koehrn FJ, Bhuskute A and Sorkin LS: Peripheral inflammation induces tumor necrosis factor dependent AMPA receptor trafficking and Akt phosphorylation in spinal cord in addition to pain behavior. Pain 149: 243-253, 2010.

19. Ren K: Emerging role of astroglia in pain hypersensitivity. Jpn Dent Sci Rev 46: 86, 2010.

20. Weyerbacher AR, Xu Q, Tamasdan C, Shin SJ and Inturrisi CE: N-Methyl-D-aspartate receptor (NMDAR) independent maintenance of inflammatory pain. Pain 148: 237-246, 2010

21. Gao X, Kim HK, Chung JM and Chung K: Reactive oxygen species (ROS) are involved in enhancement of NMDA-receptor phosphorylation in animal models of pain. Pain 131: 262-271, 2007.

22. Yowtak J, Lee KY, Kim HY, Wang J, Kim HK, Chung K and Chung JM: Reactive oxygen species contribute to neuropathic pain by reducing spinal GABA release. Pain 152: $844-852$, 2011.

23. Mollace V, Muscoli C, Rotiroti D and Nisticó G: Spontaneous induction of nitric oxide- and prostaglandin E2-release by hypoxic astroglial cells is modulated by interleukin 1 beta. Biochem Biophys Res Commun 238: 916-919, 1997.

24. Purwata TE: High TNF-alpha plasma levels and macrophages iNOS and TNF-alpha expression as risk factors for painful diabetic neuropathy. J Pain Res 4: 169-175, 2011. 